\title{
ПРЕИМУЩЕСТВА НОВОЙ СТРАТЕГИИ ДЛЯ ПРОЦЕССОРОВ CFAR ПО СРАВНЕНИЮ С МОДЕЛЬЮ НЕЙМАНА-ПИРСОНА ПРИ ОБНАРУЖЕНИИ ФЛУКТУИРУЮЩИХ ЦЕЛЕЙ, ОПИСЫВАЕМЫХ РАСПРЕДЕЛЕНИЕМ ХИ-КВАДРАТ С ЧЕТЫРЬМЯ СТЕПЕНЯМИ СВОБОДЫ
}

\author{
М. Б. ЭЛЬ МАШАД \\ Университет аль-Азхар, \\ Ezunem, Kaup, Hacep-Сumu
}

\begin{abstract}
Аннотация. Современные радары «приняли на вооружение» и используют методики адаптивной обработки для ослабления неблагоприятного влияния нежелательных мешающих отражений (clutter) и постановщиков помех (jammer). В такой ситуации алгоритмы обнаружения с постоянной вероятностью ложных тревог CFAR (Constant False Alarm Rate) играют важную роль при обнаружении флуктуирующих целей в неоднородной среде. При этом, хотя процессор с усредненной по ячейке вероятностью ложных тревог CA-CFAR (Cell-Averaging CFAR) имеет лучшие характеристики в однородной среде, методики порядковой статистики OS (Order Statistics) и усеченной по среднему значению TM (Trimmed-Mean) предложены для обеспечения устойчивых оценок порога в условиях неоднородной среды. Для одновременного использования достоинств процессора СА и процессора OS или ТМ в последнее время предложены их гибридные варианты. Они получили название моделей CAOS и CATM. Практически, частотное разнесение между несвязанными зондированиями широко распространено в реальных радиолокационных системах. Кроме того, стратегия интегрирования импульсов часто используется в радиолокационных системах для повышения отношения сигнал-шум SNR цели и улучшения характеристик обнаружения системы. Поэтому в данной работе основное внимание уделено анализу этих новых моделей в случае, когда радиолокационный приемник некогерентно интегрирует $M$-импульсы при обнаружении. Для их рабочих характеристик в условиях неоднородной среды получено выражение в замкнутой форме. Предполагается, что искомая и ложная цели описываются $\chi^{2}$ распределением с четырьмя степенями свободы их флуктуаций. Результаты моделирования, полученные авторами, показывают, что новая версия модели САТМ демонстрирует рабочую характеристику в условиях однородной среды, которая превосходит таковую для классической процедуры Неймана-Пирсона, используемую в качестве стандартной при сравнении других стратегий в области адаптивных обнаружителей.
\end{abstract}

Ключевые слова: адаптивный процессор; мешающие отражения; клаттер; помеха;, распределение хи-квадрат; модель SWIII; модель SWIV; некогерентное интегрирование; среда с групповыми целями

\section{1. ВВЕДЕНИЕ}

Радиолокационная станция (РЛС) представляет собой определенный тип систем, которые способны обнаруживать и отслеживать движущиеся или неподвижные цели. В настоящее время, помимо важности РЛС в сфере боевых действий, они также имеют многочислен- ные гражданские применения, например, управление морским и воздушным движением, метеорология и безопасность автомобильных сообщений. При этом основная функция РЛС состоит в обнаружении объектов в пределах обследуемой территории.

В большинстве случаев, по причине смешивания помеховой составляющей, известной 
Преимущества новой стратегии для процессоров СFAR по сравнению с моделью Неймана-Пирсона при обнаружении флуктуируюших целей, описываемых распределением хи-квадрат с четырьмя степенями свободы

\section{БИБЛИОГРАФИЧЕСКИЙ СПИСОК}

1. Weiner, M. A. "Detection probability for partially correlated chi-square targets," IEEE Trans. Aerospace Electronic Systems, Vol. 24, No. 4, P. 411-416, 1988. DOI: $\underline{10.1109 / 7.7183}$

2. Barkat, M.; Himonas, S. D.; Varshney, P. K. "CFAR detection for multiple target situations," IEE Proc. F - Radar Signal Processing, Vol. 136, No. 5, P. 193-209, 1989. DOI: $10.1049 /$ ip-f-2.1989.0033.

3. El Mashade, M. B. "Detection performance of the trimmed-mean CFAR processor with noncoherent integration," IEE Proc. Radar, Sonar Navig., Vol. 142, No. 1, P. 18-24, 1995. DOI: 10.1049/ip-rsn:19951626.

4. Nagle, Daniel T.; Saniie, Jafar. "Performance analysis of linearly combined order statistic CFAR detectors," IEEE Trans. Aerospace Electronic Systems, Vol. 31, No. 2, P. 522-533, 1995. DOI: 10.1109/7.381903.

5. Han, D.-S. "Detection performance of CFAR detectors based on order statistics for partially correlated chi-square targets," IEEE Trans. Aerospace Electronic Systems, Vol. 36, No. 4, P. 1423-1429, 2000. DOI: 10.11 09/7.892694.

6. Farrouki, A.; Barkat, M. "Automatic censoring CFAR detector based on ordered data variability for nonhomogeneous environments," IEE Proc. - Radar Sonar Navig., Vol. 152, No. 1, P. 43-51, 2005. DOI: 10.10 49/ip-rsn:20045006.

7. El Mashade, M. B. "Analysis of cell-averaging based detectors for $\chi^{2}$ fluctuating targets in multitarget environments," J. Electron. (China), Vol. 23, No. 6, P 853-863, 2006. DOI: 10.1007/s11767-005-0067-0.

8. Laroussi, T.; Barkat, M. "A performance comparison of two time diversity systems using CMLD-CFAR detection for partially-correlated chi-square targets and multiple target situations," Proc. of 14th European Signal Processing Conf., 4-8 Sept. 2006, Florence, Italy. IEEE, 2006, pp. 4-8, URI: https://iee explore.ieee.org/document/7071138.

9. El Mashade, M. B. "Performance analysis of OS structure of CFAR detectors in fluctuating target environments," PIER C, Vol. 2, P. 127-158, 2008. DOI: 10.2528/PIERC08022807.

10. Magaz, B.; Belouchrani, A.; Hamadouche, M. “A new adaptive linear combined CFAR detector in presence of interfering targets," PIER B, Vol. 34, P. 367-387, 2011. DOI: $10.2528 /$ PIERB1 1012603 .

11. Cai, Long; Ma, Xiaochuan; Xu, Qi; Li, Bin; Ren, Shiwei. "Performance analysis of some new CFAR detectors under clutter," J. Computers, Vol. 6, No. 6, P. 1278-1285, 2011. DOI: 10.4304/jcp.6.6.1278-1285.

12. Wang, W. Q. Radar Systems: Technology, Principles and Applications. Nova Science Publishers, Inc, 2013

13. Ivković, Dejan; Andrić, Milenko; Zrnić, Bojan. "A new model of CFAR detector," Frequenz, Vol. 68, No. 3-4, P. 125-136, 2014. DOI: $10.1515 /$ freq-2013-0087. 
14. Ivković, Dejan; Andrić, Milenko; Zrnić, Bojan. "False alarm analysis of the CATM-CFAR in presence of clutter edge," Radioengineering, Vol. 23, No. 1, P. 66-72, Apr. 2014. URI: http://www.radioeng.cz/fulltexts/2014/ 14 01_0066 0072.pdf.

15. El Mashade, M. B. "Partially-correlated $\chi 2$ targets detection analysis of GTM-adaptive processor in the presence of outliers," Int. J. Image, Graphics Signal Processing, Vol. 7, No. 12, P. 70-90, 2014. DOI: $10.5815 /$ ijigsp.2014.12.10.

16. Ivković, Dejan; Andrić, Milenko; Zrnić, Bojan; Okiljević, Predrag; Kozić, Nadica. "CATM-CFAR detector in the receiver of the software defined radar," Sci. Tech. Rev., Vol. 54, No. 4, P. 27-38, 2014. URI: http://www.vti. mod.gov.rs/ntp/rad2014/4-2014/3/e3.htm.

17. Ahmed, S. "Novel noncoherent radar pulse integration to combat noise jamming," IEEE Trans. Aerospace Electronic Systems, Vol. 51, No. 3, P. 2350-2359, 2015. DOI: 10.1109/TAES.2015.140315.
18. Ivković, Dejan; Andrić, Milenko; Zrnić, Bojan. "Detection of very close targets by fusion CFAR detectors," Sci. Tech. Rev., Vol. 66, No. 3, P. 50-57, 2016. DOI: 10.5937/STR1603050I.

19. Machado-Fernandez, J. R.; Mojena-Hernandez, N.; Bacallao-Vidal, J. C. "Evaluation of CFAR detectors performance," Iteckne, Vol. 14, No. 2, P. 170-178, 2017. DOI: $10.15332 /$ iteckne.v14i2.1772.

20. Islam, M. M.; Hossam-E-Haider, M. "Detection capability and CFAR loss under fluctuating targets of different Swerling model for various gamma parameters in RADAR," Int. J. Advanced Computer Science Applications, Vol. 9, No. 2, P. 90-93, 2018. DOI: 10.145 69/IJACSA.2018.090214.

21. El Mashade, M. B. "Heterogeneous performance analysis of the new model of CFAR detectors for partially-correlated $\chi^{2}$-targets," J. Systems Engineering Electronics, Vol. 29, No. 1, P. 1-17, 2018. DOI: 10.216 29/JSEE.2018.01.01.

Поступила в редакцию ? По-сле переработки ? 